\title{
ESTUDO EXPERIMENTAL E TEÓRICO DA REDUÇÃO DE BASES DE SCHIFF DERIVADAS DA 3,3- DIFENILPROPILAMINA
}

\author{
Andressa Esteves-Souza, Aurea Echevarria* e Carlos Mauricio R. Sant'Anna \\ Departamento de Química, Universidade Federal Rural do Rio de Janeiro, Antiga Estrada Rio - São Paulo, km 47, \\ 23851-970 Seropédica - RJ \\ Maria da Graça Nascimento \\ Departamento de Química, Universidade Federal de Santa Catarina, CP 476, 88040-900 Florianópolis - SC
}

Recebido em 10/4/03; aceito em 24/7/03

\begin{abstract}
EXPERIMENTAL AND THEORETICAL STUDY OF THE REDUCTION OF SCHIFF BASES DERIVATED FROM 3,3DIPHENYLPROPILAMINE. A series of seven Schiff bases have been synthesized from 3,3-diphenylpropilamine and substituted benzaldehydes. These imines were treated with $\mathrm{NaBH}_{4}$ in ethanol affording the corresponding amines in $98-55 \%$ yields. A molecular modeling study was performed with the Schiff bases in order to compare the theoretical parameters with the experimental results. The theoretical parameters were obtained by AM1 and PM3 semi-empirical methods. The analysis of charge, electron densities and LUMO coefficients suggested that the most favorable interactions should occur with Schiff bases containing electrondonating groups, in accordance with experimental yields, showing that the higher reactivity is due to higher electrophilic character of imine carbons.
\end{abstract}

Keywords: Schiff bases; 3,3-diphenylpropylamines; semi-empirical methods.

\section{INTRODUÇÃO}

As bases de Schiff são iminas provenientes da condensação de substâncias carbonílicas com aminas, sendo importantes intermediários envolvidos em diversas transformações enzimáticas ${ }^{1-4}$. Os mecanismos, principalmente os de formação e hidrólise já são bem conhecidos ${ }^{5}$, mas continuam sendo objeto de recentes investigações ${ }^{6,7}$.

Em contraste aos extensivos estudos de reatividade dos sistemas carbonílicos, existem relativamente poucos sobre os sistemas imínicos análogos. Apesar de haver tanto nas azometidinas quanto nos compostos carbonílicos um caráter de insaturação heteropolar, as diferenças eletrônicas entre o nitrogênio e o oxigênio causam variações marcantes nos níveis de reatividade ${ }^{8}$. As iminas são espécies reativas susceptíveis à adição de reagentes nucleofílicos, podendo assim ser utilizadas como intermediários químicos ${ }^{9}$. Recentemente, foi relatada a obtenção de heterociclos como a bis-pirazolopiridina e bisbenzilideneaminas a partir de bases de Schiff derivadas do 5-aminopirazol $^{10}$.

As bases de Schiff também têm sido sintetizadas e estudadas devido à possibilidade de sua utilização como cristais líquidos ${ }^{11}$, pois o grupamento $\mathrm{CH}=\mathrm{N}$ - estabelece uma ponte para a transmissão dos efeitos eletrônicos entre os anéis aromáticos, favorecendo a planaridade do sistema e conferindo propriedades mesogênicas ${ }^{12}$.

Além disso, as bases de Schiff têm apresentado significativa atividade biológica, dentre elas antifúngica, especialmente contra o fungo filamentoso Epidermophyton floacosum ${ }^{13}$, antimicrobiana ${ }^{14}$, citotóxica e antitumoral ${ }^{7,15,16}$.

Atualmente, a investigação sobre a estrutura molecular, antes só realizada por espectroscopia experimental e cristalografia de raios$\mathrm{X}$, vem sendo realizada também por métodos computacionais. A opção por estes métodos tem sido encorajada pelos avanços da tecnologia computacional e pelo seu baixo custo.

*e-mail: echevarr@ufrrj.br
A evolução dos cálculos envolvendo orbitais moleculares resultou em dois tipos de métodos, chamados ab initio e semi-empíricos. Nos métodos $a b$ initio, a equação de Schrödinger é resolvida sem a aplicação de parâmetros empíricos. No entanto, os cálculos com esses métodos são extremamente lentos e a sua aplicação rotineira para sistemas muito complexos, com muitos átomos, é inviável. Em comparação, simplificações introduzidas nos métodos semi-empíricos tornam viável o cálculo, em computadores de baixo custo, de sistemas moleculares com várias centenas de orbitais ${ }^{17}$. Estas simplificações incluem a consideração apenas dos elétrons de valência, a restrição do conjunto de bases à representação mínima e a negligência, em graus variáveis, do recobrimento dos orbitais atômicos localizados em átomos diferentes ${ }^{18,19}$.

Nos métodos desenvolvidos por Dewar e colaboradores, parâmetros empíricos foram introduzidos na busca da melhor correlação com dados experimentais que incluem, por exemplo, geometrias de equilíbrio, calores de formação, potenciais de ionização e momentos de dipolo ${ }^{17,20}$.

Resultados satisfatórios foram obtidos para várias propriedades, mas deve-se ter em mente que os resultados são melhores quanto mais relacionados são os dados experimentais com aqueles que se deseja calcular ${ }^{21}$.

Neste trabalho é apresentada a síntese de sete bases de Schiff da série N-p-X-benziliden-N-3,3-difenilpropilaminas (3a-g), a obtenção das aminas correspondentes através da redução por $\mathrm{NaBH}_{4}$ e o estudo sobre a reatividade das bases de Schiff frente à reação de redução por hidreto, utilizando parâmetros teóricos obtidos através dos métodos semi-empíricos AM1 e PM3, comparando-os com dados experimentais de rendimentos.

\section{RESULTADOS E DISCUSSÃO}

A preparação das bases de Schiff foi realizada a partir da 3,3difenilpropilamina e os benzaldeídos para-substituídos em etanol como solvente, sob refluxo, com rendimentos de $65-92 \%$. As bases 
de Schiff obtidas foram purificadas através de recristalização em etanol e submetidas à reação de redução na presença de $\mathrm{NaBH}_{4} \mathrm{em}$ etanol sob refluxo de $3 \mathrm{~h}$ para as iminas com grupos substituintes doadores, e $6 \mathrm{~h}$ para os compostos com substituintes retiradores de elétrons. Os rendimentos obtidos para as aminas foram de 85 a $98 \%$ (Figura 1).
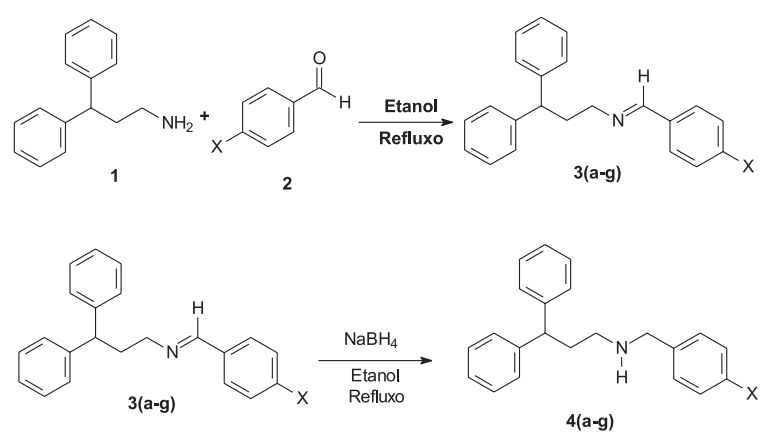

$\mathrm{X}=\mathrm{H}, \mathrm{CH}_{3}, \mathrm{~N}\left(\mathrm{CH}_{3}\right)_{2}, \mathrm{OCH}_{2} \mathrm{O}, \mathrm{Br}, \mathrm{CN}, \mathrm{NO}_{2}$

Figura 1. Rota de síntese empregada na obtenção das bases de Schiff e das aminas secundárias

Os resultados das reações de redução das N-p-X-benziliden-N3,3-difenilpropilaminas (3a-g) por transferência de hidreto foram correlacionados com os parâmetros eletrônicos obtidos através de cálculos teóricos de modelagem molecular. Os parâmetros escolhidos para a análise da correlação foram densidade eletrônica, coeficiente do LUMO (orbital de fronteira não ocupado de menor energia) e calor de formação $\left(\Delta \mathrm{H}_{\mathrm{f}}\right)$. Os valores obtidos através dos cálculos encontram-se na Tabela 1.

Analisando os valores dos parâmetros teóricos obtidos para as bases de Schiff, observou-se que os derivados com substituintes doadores de elétrons permitem ao carbono imínico um maior caráter eletrofílico, pois apresentaram menores valores de carga e densidade eletrônica. Este resultado se deve, provavelmente, ao efeito de ressonância, que concentra densidade eletrônica no carbono adjacente ao carbono imínico (Figura 2). Esta carga negativa adjacente estabilizaria o deslocamento da densidade eletrônica da ligação $\mathrm{C}=\mathrm{N}$ na direção do nitrogênio. Nota-se também que os substituintes doadores de elétrons elevam a participação do orbital $\mathrm{p}_{\mathrm{y}}$ do carbono imínico no LUMO, o que tornaria mais efetiva a interação deste carbono com um doador de elétrons num processo HOMO-LUMO.

Os substituintes retiradores de elétrons diminuem a eletrofilicidade do carbono imínico, apresentando valores maiores para carga e densidade eletrônica. Este resultado está de acordo com o anterior, pois substituintes retiradores de elétrons devem diminuir a densidade eletrônica do carbono adjacente ao carbono imínico, desestabilizando o deslocamento de carga da ligação $\mathrm{C}=\mathrm{N}$ na direção do átomo de nitrogênio. A Figura 2 indica os efeitos sugeridos, e que são coerentes com os resultados teóricos e experimentais.

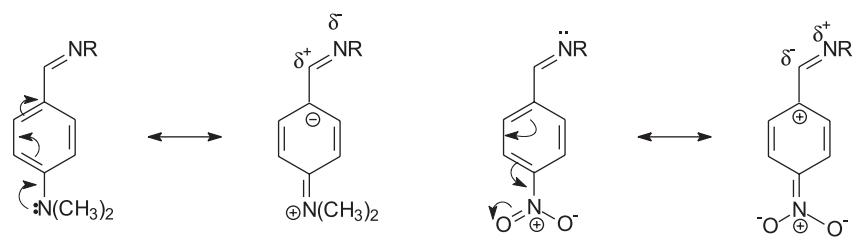

Figura 2. Influência dos efeitos eletrônicos no carbono imínico

A relação encontrada entre a natureza dos substituintes no anel ligado ao carbono imínico das moléculas e os valores de densidade eletrônica e dos coeficientes do LUMO mostrou que as bases de Schiff, com substituintes doadores são mais reativas do que as com retiradores de elétrons, o que é coerente com os resultados experimentais obtidos considerando os rendimentos dos produtos. Este resultado também está de acordo com o mecanismo da reação, pois a adição do hidreto, que ocorre na etapa lenta, é mais eficiente em centros eletrofílicos, com menor densidade eletrônica e maior participação no LUMO (Figura 3).
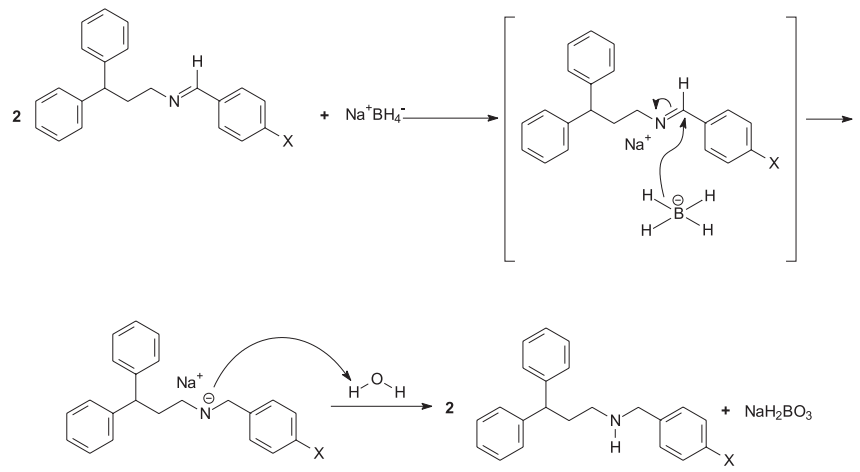

Figura 3. Mecanismo sugerido para a redução das bases de Schiff por transferência de hidreto

\section{PARTE EXPERIMENTAL}

As reações foram monitoradas por cromatografia em camada fina, em placas de alumina. Os pontos de fusão foram obtidos no aparelho MELTEMP II e não foram corrigidos. Os espectros de infravermelho

Tabela 1. Resultados obtidos nos cálculos teóricos para a série N-p-X-benziliden-N-3,3-difenilpropilaminas

\begin{tabular}{|c|c|c|c|c|c|c|c|}
\hline \multirow[t]{2}{*}{$X$} & \multicolumn{2}{|c|}{$\Delta \mathrm{H}_{\mathrm{f}}(\mathrm{Kcal})$} & \multirow[t]{2}{*}{ Rend. (\%) } & \multicolumn{2}{|c|}{$\begin{array}{l}\text { Densidade eletrônica } \\
\text { no } C_{\text {ińnico }}\end{array}$} & \multicolumn{2}{|c|}{$\begin{array}{l}\text { Coeficiente do orbital } \mathrm{p}_{\mathrm{y}} \\
\text { do } \mathrm{C}_{\text {inico }} \text { no LUMO* }\end{array}$} \\
\hline & AM1 & PM3 & & AM1 & PM3 & AM1 & PM3 \\
\hline $\mathrm{H}$ & 87,69 & 86,35 & 94 & 4,031 & 4,043 & $+0,29524$ & $+0,28391$ \\
\hline $\mathrm{CH}_{3}$ & 80,18 & 76,95 & 98 & 4,030 & 4,041 & $+0,27797$ & $-0,26312$ \\
\hline $\mathrm{N}\left(\mathrm{CH}_{3}^{3}\right)_{2}$ & 96,08 & 82,63 & 87 & 4,098 & 4,057 & $+0,03050$ & $+0,01871$ \\
\hline $\mathrm{OCH}_{2} \mathrm{O}$ & 29,15 & 24,07 & 75 & 4,027 & 4,039 & $+0,26685$ & $+0,26418$ \\
\hline $\mathrm{Br}^{2}$ & 92,61 & 94,09 & 62 & 4,032 & 4,044 & $+0,25212$ & $+0,25615$ \\
\hline $\mathrm{CN}$ & 119,60 & 121,50 & 72 & 4,036 & 4,051 & $+0,20884$ & $-0,19820$ \\
\hline $\mathrm{NO}_{2}$ & 91,47 & 77,66 & 55 & 4,044 & 4,046 & $-0,15835$ & $-0,16021$ \\
\hline
\end{tabular}

* $\mathrm{p}_{\mathrm{y}}$ é o orbital p perpendicular ao plano da ligação $\mathrm{CH}=\mathrm{N}$ - 
foram realizados em espectrômetro da Perkin-Elmer 1605 FT-IR, em pastilhas de $\mathrm{KBr}$ ou em solução de $\mathrm{CHCl}_{3}$. Os espectros de $\mathrm{RMN}$ foram obtidos nos espectrofotômetros Varian Unity-plus $(299,95 \mathrm{MHz}$ para ${ }^{1} \mathrm{H}$ e 75,42 MHz para $\left.{ }^{13} \mathrm{C}\right)$ e Bruker AC200 (200 MHz para ${ }^{1} \mathrm{H} \mathrm{e}$ $50,3 \mathrm{MHz}$ para ${ }^{13} \mathrm{C}$ ), com TMS como padrão interno, e $\mathrm{CDCl}_{3}$ ou DMSO-d 6 como solventes.

\section{Sínteses}

Procedimento geral de obtenção das bases de Schiff (3a-g)

A uma solução da 3,3-difenilpropilamina (1) $(2 \mathrm{mmol})$ foram adicionados os $p$-benzaldeídos (2a-g) $(2 \mathrm{mmol})$, em etanol. A mistura foi refluxada com agitação e a água gerada coletada em tubo Dean-Stark. O tempo de reação foi monitorado por cromatografia em camada fina. Os produtos obtidos foram filtrados e recristalizados em etanol.

\section{$N$-benziliden- $N$-3,3-difenilpropilamina (3a)}

Rendimento: $85 \%$; p. f. : $108-110^{\circ} \mathrm{C}$; IV (KBr): $v_{\max } 3026,2921-$ 2853, 1641, 1538; RMN ( $\left.\mathrm{CDCl}_{3}, 200 \mathrm{MHz}\right): \delta_{\mathrm{H}} 8,09\left(\mathrm{~s}, \mathrm{H}_{\alpha}, 1 \mathrm{H}\right)$, 3,53 (t, $\left.J=7 \mathrm{~Hz}, \mathrm{H}_{1}, 2 \mathrm{H}\right), 2,45$ (q, $\left.J=7,1 \mathrm{~Hz}, \mathrm{H}_{2}, 2 \mathrm{H}\right), 4,03(\mathrm{t}, J=7,9 \mathrm{~Hz}$, $\left.\mathrm{H}_{3}, 1 \mathrm{H}\right), 7,66-7,70\left(\mathrm{~m}, \mathrm{H}_{2}, 6^{\prime}, 2 \mathrm{H}\right), 7,37-7,40$ (m, $\left.\mathrm{H}_{3,4,5,}, 3 \mathrm{H}\right), 7,12-$ 7,26 (m, $\left.\mathrm{H}_{\text {arom }}, 10 \mathrm{H}\right)$; RMN $\left(\mathrm{CDCl}_{3}, 75 \mathrm{MHz}\right): \delta_{\mathrm{C}} 161,36\left(\mathrm{C}_{\alpha}\right), 48,61$ $\left(\mathrm{C}_{1}\right), 36,32\left(\mathrm{C}_{2}\right), 59,60\left(\mathrm{C}_{3}\right), 144,50\left(\mathrm{C}_{1}\right), 129,43\left(\mathrm{C}_{2,6}\right), 128,43$ $\left(\mathrm{C}_{3^{\prime}, 5}\right), 126,15\left(\mathrm{C}_{4}\right), 136,18\left(\mathrm{C}_{1^{\prime}}\right), 128,54\left(\mathrm{C}_{2^{\prime \prime}, 6^{\prime}}\right), 127,92\left(\mathrm{C}_{3^{\prime \prime}, 5^{\prime}}\right)$, $130,18\left(\mathrm{C}_{4},\right)$.

$N$-p-metil-benziliden- $N$-3,3-difenilpropilamina (3b)

Rendimento: $79 \%$; p. f.: $163-165^{\circ} \mathrm{C}$; IV (KBr): $v_{\max } 3023,2928-$ 2832, 1643, 1493, 859, 740; RMN ( $\left.\mathrm{CDCl}_{3}, 200 \mathrm{MHz}\right): \delta_{\mathrm{H}} 8,05$ (s, $\left.\mathrm{H}_{\alpha}, 1 \mathrm{H}\right), 3,51$ (t, $\left.J=6 \mathrm{~Hz}, \mathrm{H}_{1}, 2 \mathrm{H}\right), 2,45\left(\mathrm{q}, J=7,2 \mathrm{~Hz}, \mathrm{H}_{2}, 2 \mathrm{H}\right), 4,02$ (t, $\left.J=7,9 \mathrm{~Hz}, \mathrm{H}_{3}, 1 \mathrm{H}\right), 7,12-7,28\left(\mathrm{~m}, \mathrm{H}_{\text {arom }}, 12 \mathrm{H}\right), 7,57$ (d, $J=8,3 \mathrm{~Hz}, \mathrm{H}_{2,}, 6^{\prime}$, $2 \mathrm{H}), 2,36\left(\mathrm{~s}, \mathrm{CH}_{3}, 3 \mathrm{H}\right)$; RMN ( $\left.\mathrm{CDCl}_{3}, 75 \mathrm{MHz}\right): \delta_{\mathrm{C}} 161,27\left(\mathrm{C}_{\alpha}\right)$, $48,60\left(\mathrm{C}_{1}\right), 36,36\left(\mathrm{C}_{2}\right), 59,59\left(\mathrm{C}_{3}\right), 144,53\left(\mathrm{C}_{1}\right), 129,27\left(\mathrm{C}_{2,6}\right), 128,42$ $\left(\mathrm{C}_{3^{\prime}, 5}\right), 126,12\left(\mathrm{C}_{4}\right), 133,60\left(\mathrm{C}_{1^{\prime}}\right), 127,92\left(\mathrm{C}_{3^{\prime}, 6^{\prime}}\right), 140,74\left(\mathrm{C}_{4^{\prime}}\right), 21,45$ $\left(\underline{\mathrm{CH}}_{3}\right)$.

$N$-p-dimetilamino-benziliden- $N$-3,3-difenilpropilamina $(\mathbf{3 c})$

Rendimento: 87\%; p. f.: $108-110{ }^{\circ} \mathrm{C}$; IV (KBr): $v_{\max } 3023,2929-$ 2856, 1603, 1531, 813, 749; RMN $\left(\mathrm{CDCl}_{3}, 200 \mathrm{MHz}\right): \delta_{\mathrm{H}} 8,02(\mathrm{~s}$, $\left.\mathrm{H}_{\alpha}, 1 \mathrm{H}\right), 3,58\left(\mathrm{t}, J=6,6 \mathrm{~Hz}, \mathrm{H}_{1}, 2 \mathrm{H}\right), 2,50$ (q, $\left.J=6,6 \mathrm{~Hz}, \mathrm{H}_{2}, 2 \mathrm{H}\right), 4,10$ $\left(\mathrm{t}, J=7,6 \mathrm{~Hz}, \mathrm{H}_{3}, 1 \mathrm{H}\right), 7,30\left(\mathrm{~m}, \mathrm{H}_{\text {arom }}, 10 \mathrm{H}\right), 7,62\left(\mathrm{dd}, J_{o}=9,4 ; J_{p}=1,9\right.$ $\left.\mathrm{Hz}, \mathrm{H}_{2,, 6,}, 2 \mathrm{H}\right), 6,70\left(\mathrm{dd}, J_{0}=9,4 ; \mathrm{J}_{\mathrm{p}}=1,9 \mathrm{~Hz}, \mathrm{H}_{3,5,}, 2 \mathrm{H}\right), 3,00(\mathrm{~s}$, $\left.\mathrm{N}\left(\mathrm{CH}_{3}\right)_{2}, 6 \mathrm{H}\right) ; \mathrm{RMN}\left(\mathrm{CDCl}_{3}, 75 \mathrm{MHz}\right): \delta_{\mathrm{C}} 161,33\left(\mathrm{C}_{\alpha}\right), 48,56\left(\mathrm{C}_{1}\right)$, $36,52\left(\mathrm{C}_{2}\right), 59,42\left(\mathrm{C}_{3}\right), 144,62\left(\mathrm{C}_{1}\right), 129,37\left(\mathrm{C}_{2,6,}\right), 128,33\left(\mathrm{C}_{3,5},\right)$, $126,00\left(\mathrm{C}_{4}\right), 124,24(\mathrm{C} 1$ ") $), 127,64\left(\mathrm{C}_{2 ", 6},{ }^{\prime}\right), 111,51\left(\mathrm{C}_{3,, 5},{ }^{\prime}\right), 151,89$ $\left(\mathrm{C}_{4}{ }^{\prime \prime}\right), 40,13\left(\mathrm{~N}\left(\underline{\mathrm{CH}}_{3}\right)_{2}\right)$.

\section{$N$-3,4-metilenedioxi-benziliden- $N$-3,3-difenilpropilamina (3d)}

Rendimento: $72 \%$; p. f.: $105-107^{\circ} \mathrm{C}$; IV (KBr): $v_{\max } 3065,2924-$ 2820, 1649, 1495, 1195-1125, 819, 742; RMN (CDCl $200 \mathrm{MHz})$ : $\delta_{\mathrm{H}} 7,97\left(\mathrm{~s}, \mathrm{H}_{\alpha}, 1 \mathrm{H}\right), 3,48\left(\mathrm{t}, J=6,9 \mathrm{~Hz}, \mathrm{H}_{1}, 2 \mathrm{H}\right), 2,43\left(\mathrm{q}, J=7,2 \mathrm{~Hz}, \mathrm{H}_{2}\right.$, $2 \mathrm{H}), 4,02\left(\mathrm{t}, J=7,9 \mathrm{~Hz}, \mathrm{H}_{3}, 1 \mathrm{H}\right), 7,24\left(\mathrm{~m}, \mathrm{H}_{\text {arom }}, 10 \mathrm{H}\right), 7,32\left(\mathrm{sl}, \mathrm{H}_{2}\right.$, , $1 \mathrm{H}), 7,01\left(\mathrm{~d}, J=7,9 \mathrm{~Hz}, \mathrm{H}_{5}, 1 \mathrm{H}\right), 6,80\left(\mathrm{~d}, J=7,9 \mathrm{~Hz}, \mathrm{H}_{6,}, 1 \mathrm{H}\right), 5,98(\mathrm{~s}$, $\left.\mathrm{OC} \underline{H}_{2} \mathrm{O}, 2 \mathrm{H}\right)$; RMN $\left(\mathrm{CDCl}_{3}, 75 \mathrm{MHz}\right): \delta_{\mathrm{C}} 160,51\left(\mathrm{C}_{\alpha}\right), 48,59\left(\mathrm{C}_{1}\right)$, $36,78\left(\mathrm{C}_{2}\right), 59,32\left(\mathrm{C}_{3}\right), 144,52\left(\mathrm{C}_{1}\right), 128,43\left(\mathrm{C}_{2},\right), 127,92\left(\mathrm{C}_{3}\right), 126,15$ $\left(\mathrm{C}_{4}\right), 131,03\left(\mathrm{C}_{1},\right), 106,47\left(\mathrm{C}_{2^{\prime}}\right), 148,19\left(\mathrm{C}_{3^{\prime}}\right), 153,28\left(\mathrm{C}_{4^{\prime}}\right), 108,02$ $\left(\mathrm{C}_{5},\right), 124,16\left(\mathrm{C}_{6}\right), 101,38\left(\mathrm{OCH}_{2} \mathrm{O}\right)$.

$N$-p-bromo-benziliden- $N$-3,3-difenilpropilamina (3e)

Rendimento: 65\%; p. f.: 99-102 ${ }^{\circ} \mathrm{C}$; IV (KBr): $v_{\max } 3021,2929-$ 2835, 1639, 1586, 819, 743; RMN $\left(\mathrm{CDCl}_{3}, 200 \mathrm{MHz}\right): \delta_{\mathrm{H}} 8,04(\mathrm{~s}$, $\left.\mathrm{H}_{\mathrm{a}}, 1 \mathrm{H}\right), 3,48\left(\mathrm{t}, J=6,9 \mathrm{~Hz}, \mathrm{H}_{1}, 2 \mathrm{H}\right), 2,43\left(\mathrm{q}, J=7,2 \mathrm{~Hz}, \mathrm{H}_{2}, 2 \mathrm{H}\right), 4,02$ (t, $\left.J=7,9 \mathrm{~Hz}, \mathrm{H}_{3}, 1 \mathrm{H}\right), 7,14-7,31\left(\mathrm{~m}, \mathrm{H}_{\text {arom }}, 12 \mathrm{H}\right), 7.53\left(\mathrm{~m}, \mathrm{H}_{3^{\prime \prime}, 5,}, 2 \mathrm{H}\right)$; $\mathrm{RMN}\left(\mathrm{CDCl}_{3}, 75 \mathrm{MHz}\right): \delta_{\mathrm{C}} 160,09\left(\mathrm{C}_{\alpha}\right), 48,66\left(\mathrm{C}_{1}\right), 36,23\left(\mathrm{C}_{2}\right)$, 59,62 ( $\left.\mathrm{C}_{3}\right), 144,43\left(\mathrm{C}_{1}\right), 129,41\left(\mathrm{C}_{2,6}\right), 127,90\left(\mathrm{C}_{3}, 5,{ }^{,}\right), 126,21\left(\mathrm{C}_{4}\right)$, $135,07\left(\mathrm{C}_{1,}\right), 128,46\left(\mathrm{C}_{2,, 6^{\prime}}\right), 131,79\left(\mathrm{C}_{3^{\prime \prime},{ }^{\prime}}\right), 124,89\left(\mathrm{C}_{4^{\prime}}\right)$.

\section{$N$-p-ciano-benziliden- $N$-3,3-difenilpropilamina $(\mathbf{3 f})$}

Rendimento: $80 \%$; p. f.: $78-80{ }^{\circ} \mathrm{C}$; IV (KBr): $v_{\max } 3056,2934-$ 2844, 2228, 1644, 1550, 836, 750; RMN ( $\left.\mathrm{CDCl}_{3}, 200 \mathrm{MHz}\right): \delta_{\mathrm{H}}$ $8,11\left(\mathrm{~s}, \mathrm{H}_{\alpha}, 1 \mathrm{H}\right), 3,57$ (t, 6,9Hz, H1, 2H), 2,45 (q, J=7,1Hz, H2, 2H), 4,01 (t, J=7,8Hz, H3, 1H), 7,10-7,26 (m, $\left.\mathrm{H}_{\text {arom }}, 10 \mathrm{H}\right), 7,77$ (d, $\left.J=6,7 \mathrm{~Hz}, \mathrm{H}_{2,, 6}, 2 \mathrm{H}\right), 7,66\left(\mathrm{~d}, J=6,7 \mathrm{~Hz}, \mathrm{C}_{3,},{ }^{\prime \prime}, 2 \mathrm{H}\right) ; \mathrm{RMN}\left(\mathrm{CDCl}_{3}, 75\right.$ $\mathrm{MHz}): \delta_{\mathrm{C}} 159,41\left(\mathrm{C}_{\alpha}\right), 48,68\left(\mathrm{C}_{1}\right), 36,13\left(\mathrm{C}_{2}\right), 59,76\left(\mathrm{C}_{3}\right), 144,29$ $\left(\mathrm{C}_{1}\right), 128,50\left(\mathrm{C}_{2}\right), 128,40\left(\mathrm{C}_{3}\right), 126,27\left(\mathrm{C}_{4}\right), 139,98\left(\mathrm{C}_{1},{ }^{\prime}\right), 127,86$ $\left(\mathrm{C}_{2^{\prime \prime}, 6^{\prime}}\right), 127,47\left(\mathrm{C}_{3^{\prime \prime}, 5^{\prime}}\right), 129,85\left(\mathrm{C}_{4^{\prime}}\right), 118,53(\underline{\mathrm{CN}})$.

\section{$N$-p-nitro-benziliden- $N$-3,3-difenilpropilamina $(\mathbf{3 g})$}

Rendimento: $92 \%$; p. f.: $96-98{ }^{\circ} \mathrm{C}$; IV (KBr): $v_{\max } 3058,2933-$ 2835, 1645, 1598, 832, 747; RMN ( $\left.\mathrm{CDCl}_{3}, 200 \mathrm{MHz}\right): \delta_{\mathrm{H}} 8,26(\mathrm{~s}$, $\left.\mathrm{H}_{\alpha}, 1 \mathrm{H}\right), 3,59$ (t, $\left.J=6,9 \mathrm{~Hz}, \mathrm{H}_{1}, 2 \mathrm{H}\right), 2,47$ (q, $\left.J=7,9 \mathrm{~Hz}, \mathrm{H}_{2}, 2 \mathrm{H}\right), 4,03$ (t, $\left.J=7,9 \mathrm{~Hz}, \mathrm{H}_{3}, 1 \mathrm{H}\right), 7,14-7,27\left(\mathrm{~m}, \mathrm{H}_{\text {arom }}, 10 \mathrm{H}\right), 8,19(\mathrm{~d}, J=10,4 \mathrm{~Hz}$, $\left.\mathrm{H}_{2,, 6}, 2 \mathrm{H}\right), 7,83\left(\mathrm{~d}, J=10,4 \mathrm{~Hz}, \mathrm{C}_{3,}, 5^{\prime}, 2 \mathrm{H}\right) ; \mathrm{RMN}\left(\mathrm{CDCl}_{3}, 75 \mathrm{MHz}\right)$; $\delta_{\mathrm{C}} 158,97\left(\mathrm{C}_{\alpha}\right), 48,68\left(\mathrm{C}_{1}\right), 36,09\left(\mathrm{C}_{2}\right), 59,84\left(\mathrm{C}_{3}\right), 144,25\left(\mathrm{C}_{1}\right)$, $128,61\left(\mathrm{C}_{2,6},{ }^{\alpha}\right), 128,49\left(\mathrm{C}_{3,5},\right), 126,25\left(\mathrm{C}_{4}\right), 141,61\left(\mathrm{C}_{1},{ }^{\prime}\right), 127,82$ $\left(\mathrm{C}_{2^{\prime \prime}, 6^{\prime}}\right), 148,84\left(\mathrm{C}_{3^{\prime \prime}, 5^{\prime \prime}}\right), 123,80\left(\mathrm{C}_{4^{\prime \prime}}\right)$.

\section{Procedimento geral para obtenção das aminas ${ }^{22}$ (4a-g)}

A uma solução etanólica das bases de Schiff (3a-g) $(2 \mathrm{mmol})$ foram adicionados lentamente, em banho de gelo e com agitação $\mathrm{NaBH}_{4}(2 \mathrm{mmol})$. A mistura foi refluxada durante 3-6 h, dependendo da imina a ser reduzida. Após este tempo, o solvente foi evaporado e água foi adicionada $(2 \mathrm{~mL})$. A extração do produto foi realizada com $\mathrm{CHCl}_{3}(3 \times 2 \mathrm{~mL})$. A fase orgânica foi seca com $\mathrm{Na}_{2} \mathrm{SO}_{4}$, e após evaporação do solvente, obteve-se o produto, que foi purificado em coluna cromatográfica flash (gel de sílica 60, 35-70 mesh) com $\mathrm{CHCl}_{3}$ como eluente.

\section{$N$-[3,3-difenilpropil]-N-benzilamina (4a)}

Rendimento: 94\%; Índice de refração: 1,444; IV (NaCl): $v_{\max }$ 3408, 3025, 2923-2851, 1492, 1115, 744; RMN (CDCl, $200 \mathrm{MHz})$ : $\delta_{\mathrm{H}} 3,71\left(\mathrm{~s}, \mathrm{H}_{\alpha}, 2 \mathrm{H}\right), 2,61\left(\mathrm{t}, J=7,5 \mathrm{~Hz}, \mathrm{H}_{1}, 2 \mathrm{H}\right), 2,26\left(\mathrm{q}, J=7 \mathrm{~Hz}, \mathrm{H}_{2}\right.$, $2 \mathrm{H}), 4,02\left(\mathrm{t}, J=7,8 \mathrm{~Hz}, \mathrm{H}_{3}, 1 \mathrm{H}\right), 7,15-7,30\left(\mathrm{~m}, \mathrm{H}_{\text {arom }}, 15 \mathrm{H}\right), 1,55(\mathrm{~s}$, $\mathrm{NH}, 1 \mathrm{H}) ; \mathrm{RMN}\left(\mathrm{CDCl}_{3}, 75 \mathrm{MHz}\right): \delta_{\mathrm{C}} 49,01\left(\mathrm{C}_{\alpha}\right), 47,71\left(\mathrm{C}_{1}\right), 35,82$ $\left(\mathrm{C}_{2}\right), 53,89\left(\mathrm{C}_{3}\right), 144,77\left(\mathrm{C}_{1}\right), 128,36\left(\mathrm{C}_{2,6}\right), 127,82\left(\mathrm{C}_{3,5},{ }^{,}\right), 126,15$ $\left(\mathrm{C}_{4}\right), 140,31\left(\mathrm{C}_{1,}\right), 128,43\left(\mathrm{C}_{2^{\prime \prime}, 6^{\prime}}\right), 128,06\left(\mathrm{C}_{3^{\prime \prime}, 5^{\prime}}\right), 126,88\left(\mathrm{C}_{4^{\prime}}\right)$

\section{$N$-[3,3-difenilpropil]- $N$-[p-metil-benzil]-amina $(\mathbf{4 b})$}

Rendimento: 98\%; Índice de refração: volátil; IV (NaCl): $v_{\max }$ 3322, 2925-2822, 1493, 804, 749; RMN ( $\left.\mathrm{CDCl}_{3}, 200 \mathrm{MHz}\right): \delta_{\mathrm{H}} 3,6$ (s, $\left.\mathrm{H}_{\alpha}, 2 \mathrm{H}\right), 2,61\left(\mathrm{t}, J=7,5 \mathrm{~Hz}, \mathrm{H}_{1}, 2 \mathrm{H}\right), 2,26\left(\mathrm{q}, J=6,8 \mathrm{~Hz}, \mathrm{H}_{2}, 2 \mathrm{H}\right)$,

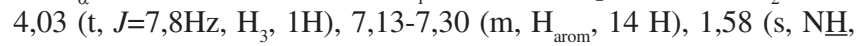
$1 \mathrm{H}), 2,33\left(\mathrm{~s}, \mathrm{C}_{3}, 3 \mathrm{H}\right) ; \mathrm{RMN}\left(\mathrm{CDCl}_{3}, 75 \mathrm{MHz}\right): \delta_{\mathrm{C}} 48,92\left(\mathrm{C}_{\alpha}\right), 47,59$ $\left(\mathrm{C}_{1}\right), 35,73\left(\mathrm{C}_{2}\right), 53,51\left(\mathrm{C}_{3}\right), 144,72\left(\mathrm{C}_{1}\right), 128,95\left(\mathrm{C}_{2,6},{ }^{\prime}\right), 128,36$ $\left(\mathrm{C}_{3,5},\right), 126,06\left(\mathrm{C}_{4}\right), 137,19\left(\mathrm{C}_{1,}\right), 127,94\left(\mathrm{C}_{2^{\prime}, 6^{\prime}}\right), 127,73\left(\mathrm{C}_{3^{\prime \prime}, 5^{\prime}}\right)$, $136,32\left(\mathrm{C}_{4},\right), 21,01\left(\underline{\mathrm{CH}}_{3}\right)$.

\section{$\mathrm{N}$-[3,3-difenilpropil]- $\mathrm{N}$-[p-dimetilamino-benzil]-amina (4c)}

Rendimento: 65\%; Índice de refração: volátil; IV $(\mathrm{NaCl}): v_{\max }$ 3306, 3058-3024, 2928-2854, 1491, 1125, 806, 750; RMN ( $\mathrm{CDCl}_{3}$, $200 \mathrm{MHz}): \delta_{\mathrm{H}} 3,65\left(\mathrm{~s}, \mathrm{H}_{\alpha}, 2 \mathrm{H}\right), 2,62(\mathrm{t},=8 \mathrm{~Hz}, \mathrm{H} 1,2 \mathrm{H}), 2,33$ $(J=6,5 \mathrm{~Hz}, \mathrm{H} 2,2 \mathrm{H}), 4,01$ (t, $J=6,5 \mathrm{~Hz}, \mathrm{H} 3,1 \mathrm{H}), 7,13-7,26$ (m, $\mathrm{H}_{\text {arom? }}$, $12 \mathrm{H}), 6,67$ (d, J=8,7Hz, $\left.\mathrm{H}_{3,, 5}, 1 \mathrm{H}\right), 2,55(\mathrm{~s}, \mathrm{~N} \underline{\mathrm{H}}, 1 \mathrm{H}), 2,91(\mathrm{~s}$, $\left.\mathrm{N}\left(\mathrm{CH}_{3}\right)_{2}, 3 \mathrm{H}\right) ; \mathrm{RMN}\left(\mathrm{CDCl}_{3}, 75 \mathrm{MHz}\right): \delta_{\mathrm{C}} 48,95\left(\mathrm{C}_{\alpha}\right), 47,29\left(\mathrm{C}_{1}\right)$, $35,38\left(\mathrm{C}_{2}\right), 53,06\left(\mathrm{C}_{3}\right), 144,65\left(\mathrm{C}_{1}\right), 128,39\left(\mathrm{C}_{2,6}\right), 127,77\left(\mathrm{C}_{3,5},{ }^{,}\right)$, 
$126,09\left(\mathrm{C}_{4^{\prime}}\right), 127,42\left(\mathrm{C}_{1^{\prime}}\right), 129,15\left(\mathrm{C}_{2^{\prime}, 6^{\prime}}\right), 112,62\left(\mathrm{C}_{3^{\prime \prime}, 5^{\prime}}\right), 149,82\left(\mathrm{C}_{4^{\prime}}\right)$, $40,68\left(\mathrm{~N}\left(\mathrm{CH}_{3}\right)_{2}\right)$.

$N$-[3,3-difenilpropil]-N-[3,4-metilenedioxi-benzil]-amina (4d)

Rendimento: 75\%; Índice de refração: 1,450; IV $(\mathrm{NaCl}): v_{\text {max }}$ $3643,3059-3026,2926,1493,1189,1107,809,750 ; \mathrm{RMN}\left(\mathrm{CDCl}_{3}\right.$, $200 \mathrm{MHz}): \delta_{\mathrm{H}} 3,62\left(\mathrm{~s}, \mathrm{H}_{\alpha}, 2 \mathrm{H}\right), 2,58\left(\mathrm{t}, J=7,5 \mathrm{~Hz}, \mathrm{H}_{1}, 2 \mathrm{H}\right), 2,26(\mathrm{q}$, $\left.J=7,6 \mathrm{~Hz}, \mathrm{H}_{2}, 2 \mathrm{H}\right), 4,02$ (t, $\left.J=7,7 \mathrm{~Hz}, \mathrm{H}_{3}, 1 \mathrm{H}\right), 7,13-7,30\left(\mathrm{~m}, \mathrm{H}_{\text {arom }}\right.$, $10 \mathrm{H}), 6,77\left(\mathrm{~m}, \mathrm{H}_{2,}, 1 \mathrm{H}\right), 6,71\left(\mathrm{~m}, \mathrm{H}_{5,}, 1 \mathrm{H}\right), 1,72(\mathrm{~s}, \mathrm{~N} \underline{\mathrm{H}}, 1 \mathrm{H}), 5,92(\mathrm{~s}$, $\left.\mathrm{OCH}_{2} \mathrm{O}, 2 \mathrm{H}\right)$; NMR $\left(\mathrm{CDCl}_{3}, 75 \mathrm{MHz}\right): \delta_{\mathrm{C}} 48,86\left(\mathrm{C}_{\alpha}\right), 47,29\left(\mathrm{C}_{1}\right)$, $35,52\left(\mathrm{C}_{2}\right), 53,42\left(\mathrm{C}_{3}\right), 144,62\left(\mathrm{C}_{1}\right), 128,35\left(\mathrm{C}_{2,6}\right), 127,70\left(\mathrm{C}_{3,5},{ }^{\prime}\right)$, $126,07\left(\mathrm{C}_{4}\right), 133,86\left(\mathrm{C}_{1}\right), 108,58\left(\mathrm{C}_{2^{\prime}}\right), 147,53\left(\mathrm{C}_{3^{\prime}}\right), 146,38\left(\mathrm{C}_{4^{\prime}}\right)$, $107,92\left(\mathrm{C}_{5^{\prime \prime}}\right), 121,12\left(\mathrm{C}_{6^{\prime}}\right), 110,74\left(\mathrm{OCH}_{2} \mathrm{O}\right)$.

\section{$N$-[3,3-difenilpropil]- $N$-[p-bromo-benzil]-amina $(\mathbf{4 e})$}

Rendimento: 62\%; Índice de refração: volátil; IV $(\mathrm{NaCl}): v_{\max }$ 3322, 3059-3026, 2927-2846, 1489, 1116, 819, 749; RMN (CDCl $200 \mathrm{MHz}): \delta_{\mathrm{H}} 3,62\left(\mathrm{~s}, \mathrm{H}_{\alpha}, 2 \mathrm{H}\right), 2,55\left(\mathrm{t}, J=6,3 \mathrm{~Hz}, \mathrm{H}_{1}, 2 \mathrm{H}\right), 2,23(\mathrm{q}$, $\left.J=7,7 \mathrm{~Hz}, \mathrm{H}_{2}, 2 \mathrm{H}\right), 4,00\left(\mathrm{t}, J=7,8 \mathrm{~Hz}, \mathrm{H}_{3}, 1 \mathrm{H}\right), 7,06-7,41$ (m, $\mathrm{H}_{\text {arom }}, 14$ $\mathrm{H}), 1,55(\mathrm{~s}, \mathrm{~N} \underline{\mathrm{H}}, 1 \mathrm{H}) ; \mathrm{RMN}\left(\mathrm{CDCl}_{3}, 75 \mathrm{MHz}\right): \delta_{\mathrm{C}} 48,74\left(\mathrm{C}_{\alpha}\right), 47,37$ $\left(\mathrm{C}_{1}\right), 35,60\left(\mathrm{C}_{2}\right), 52,90\left(\mathrm{C}_{3}\right), 144,53\left(\mathrm{C}_{1}\right), 128,29\left(\mathrm{C}_{2,6},{ }^{\prime}\right), 127,61$ $\left(\mathrm{C}_{3^{\prime}, 5}\right), 126,02\left(\mathrm{C}_{4}\right), 139,19\left(\mathrm{C}_{1}\right), 129,57\left(\mathrm{C}_{2^{\prime \prime}, 6^{\prime}}\right), 131,16\left(\mathrm{C}_{3^{\prime \prime}, 5^{\prime}}\right)$, $120,40\left(\mathrm{C}_{4}\right)$.

\section{$N$-[3,3-difenilpropil]- $N$-[p-ciano-benzil]-amina (4f)}

Produto: 72\%; Índice de refração: volátil; IV $(\mathrm{NaCl}): v_{\max } 3314$, 3058-3026, 2929-2854, 2226, 1494, 1113, 818, 747; RMN ( $\mathrm{CDCl}_{3}$, $200 \mathrm{MHz}): \delta_{\mathrm{H}} 3,78\left(\mathrm{~s}, \mathrm{H}_{\alpha}, 2 \mathrm{H}\right), 2,65\left(\mathrm{t}, J=6,9 \mathrm{~Hz}, \mathrm{H}_{1}, 2 \mathrm{H}\right), 2,28(\mathrm{q}$, $\left.J=5,3 \mathrm{~Hz}, \mathrm{H}_{2}, 2 \mathrm{H}\right), 4,03$ (t, $\left.J=4,1 \mathrm{~Hz}, \mathrm{H}_{3}, 1 \mathrm{H}\right), 7,14-7,37$ (m, $\mathrm{H}_{\text {arom }}, 14$ $\mathrm{H})$, não observado $(\mathrm{N} \underline{\mathrm{H}})$; $\mathrm{RMN}\left(\mathrm{CDCl}_{3}, 75 \mathrm{MHz}\right): \delta_{\mathrm{C}} 48,89\left(\mathrm{C}_{\alpha}\right)$, $47,57\left(\mathrm{C}_{1}\right), 35,65\left(\mathrm{C}_{2}\right), 53,22\left(\mathrm{C}_{3}\right), 144,43\left(\mathrm{C}_{1}\right), 128,48\left(\mathrm{C}_{2^{\prime}, 6}\right), 127,71$ $\left(\mathrm{C}_{3^{\prime}, 5^{\prime}}\right), 126,24\left(\mathrm{C}_{4}\right), 145,80\left(\mathrm{C}_{1^{\prime \prime}}\right), 132,12\left(\mathrm{C}_{2^{\prime \prime}, 6^{\prime \prime}}\right), 128,48\left(\mathrm{C}_{3^{\prime \prime}, 5^{\prime}}\right)$.

\section{$\mathrm{N}$-[3,3-difenilpropil]- $\mathrm{N}$-[p-nitro-benzil]-amina $(\mathbf{4 g})$}

Rendimento: 55\%; Índice de refração: 1,456; IV (NaCl): $v_{\max }$ 3332, 3059-3026, 2929-2850, 1495, 845, 748; RMN $\left(\mathrm{CDCl}_{3}, 200\right.$ $\mathrm{MHz}): \delta_{\mathrm{H}} 3,81\left(\mathrm{~s}, \mathrm{H}_{\alpha}, 2 \mathrm{H}\right), 2,59\left(\mathrm{t}, J=7 \mathrm{~Hz}, \mathrm{H}_{1}, 2 \mathrm{H}\right), 2,30(\mathrm{q}, J=7,3 \mathrm{~Hz}$, $\left.\mathrm{H}_{2}, 2 \mathrm{H}\right), 4,06$ (t, $\left.J=7,8 \mathrm{~Hz}, \mathrm{H}_{3}, 1 \mathrm{H}\right), 7,16-7,29$ (m, $\left.\mathrm{H}_{\text {arom }}, 10 \mathrm{H}\right), 8,05$

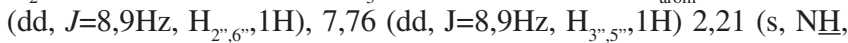
$1 \mathrm{H})$; RMN (CDCl $3,75 \mathrm{MHz}): \delta_{\mathrm{C}} 48,80\left(\mathrm{C}_{\alpha}\right), 48,65\left(\mathrm{C}_{1}\right), 36,06\left(\mathrm{C}_{2}\right)$, $52,95\left(\mathrm{C}_{3}\right), 141,56\left(\mathrm{C}_{1}\right), 128,46\left(\mathrm{C}_{2,6},{ }^{\prime}\right), 127,79\left(\mathrm{C}_{3,5},{ }^{\circ}\right), 126,22\left(\mathrm{C}_{4}\right)$, $144,23\left(\mathrm{C}_{1},\right), 128,58\left(\mathrm{C}_{2,}, 6^{\prime}\right), 123,76\left(\mathrm{C}_{3,, 5},{ }^{\prime}\right), 144,23\left(\mathrm{C}_{4,}\right)$.

\section{Modelagem molecular}

O programa escolhido para realizar o estudo foi o MOPAC 6.0 para Windows 95 e os métodos semi-empíricos utilizados foram AM1 e PM3, em uma unidade PC Pentium $300 \mathrm{MHz}$. As estruturas foram otimizadas até uma norma de gradiente $0,08 \mathrm{kcal} / \mathrm{rad}$. Os coeficientes dos orbitais atômicos moleculares foram obtidos com a palavrachave VECTORS.

\section{CONCLUSÕES}

Os resultados obtidos nos cálculos de modelagem molecular sugerem uma explicação para os dados experimentais obtidos neste trabalho: o maior caráter eletrofílico do $\mathrm{C}_{\alpha}$ seria o fator determinante dos maiores rendimentos obtidos na reação de redução, para os derivados com grupos doadores de elétrons.

\section{AGRADECIMENTOS}

Este trabalho foi realizado com suporte financeiro do $\mathrm{CNPq}$, CAPES e dos Programas de Pós-graduação da UFRRJ e UFSC.

\section{REFERÊNCIAS}

1. Williams, S. D.; Danid, S. S.; Biochem.1999, 38, 15417.

2. Dodson, M. L.; Michaels, M. L.; Lloyd, S. R.; J. Biol. Chem. 1994, 269, 32709 .

3. Snell, E.E.; Mari, S. J. D.; The Enzymes-Kinetics and Mechanism: Schiff Bases Intermediates in Enzyme Catalysis, $3^{\text {th }}$ ed., Academic Press: New York, 1970, vol. II.

4. Okuyama, T.; Majamatsu, H.; Kitano, M.; Fueno, T.; J. Org. Chem. 1986, $51,1516$.

5. Jencks, W. P.; Prog. Phys. Org. Chem. 1964, 2, 63.

6. Wang, M.; McIntee, E. J.; Cheng, G.; Shi, Y.; Villala, P. W.; Hencht, S. S.; Chem. Res. Toxicol. 2001, 14, 423.

7. Ren, S.; Wang, R.; Komatsu, K.; Bonaz-Krause, P.; Zyrianov, Y.; McKennna, C. E.; Csiphe, C.; Tokes, Z. A.; Lien, E. J.; J. Med. Chem. 2002, 45, 410 .

8. Patai, S.; The Chemistry of Amides, Wiley: New York, 1975.

9. Pilli, R. A.; Russowsky, D.; Petersen, R. Z.; Godoi, M. N.; Tetrahedron Lett. 2000, 41, 9939.

10. Esteves-Souza, A.; Echevarria, A.; Vencato, I.; Jimeno, M. L.; Elguero, J.; Tetrahedron 2001, 57, 6147.

11. Gallardo, H.; Parra, M.; Zúniga, C.; Hidalgo, P.; Vergara, J.; Hernández, S.; Liq. Cryst. 2001, 28, 1659.

12. Claramunt, R. M.; Forfar, I.; Cabildo, P.; Lafuente, J.; Berberá, J.; Elguero, J.; Heterocycles 1999, 51, 751 .

13. Echevarria, A.; Nascimento, M. G.; Gerônimo, V.; Miller, J.; Giesbrecht, A.; J. Braz. Chem. Soc. 1999, 10, 60.

14. Perry, B. F.; Beezer, A. E.; Miles, R.J.; Smith, B. V.; Miller, J.; Nascimento, M. G.; Microbios 1986, 45, 181.

15. Dimmock, J. R.; Curr. Med. Chem. 1997, 4, 22.

16. Dimmock, J. R.; Advikolanu, K. M.; Scott, H. E.; Duffy, M. F.; Reid, R. S.; Quail, J. W.; Jia, Z.; Hickie, R. A.; Allen, T. M.; Rutledge, J. M.; Tempest, M. L.; Oreski, A. B.; J. Pharm. Sci. 1992, 81, 1147.

17. Clark, T. A.; A Handbook of Computational Chemistry, Wiley: New York, 1985; Hirst, D. M.; A Computational Approach to Chemistry, Blackwell Scientific: Oxford, 1990.

18. Stewart, J. P. J.; J. Comput. Chem. 1989, 2, 209.

19. Stewart, J. P. J.; MOPAC, A Semiempirical Molecular Orbital Program, QCPE, 1983, p. 455.

20. Dewar, M. J. S.; Zoebisch, E. G.; Eamonn, F. H.; Stewart, J. P. J.; J. Am. Chem. Soc. 1985, 13, 3902.

21. Sant' Anna, C. M. R.; Souza, V. P.; Quim. Nova 2001, 24, 583.

22. Esteves-Souza, A.; Tese de Mestrado, Universidade Federal Rural do Rio de Janeiro, Brasil, 1999 